\title{
Finiteness of arithmetic hyperbolic reflection groups
}

\author{
Ian $\mathrm{Agol}^{1}$, Mikhail Belolipetsky ${ }^{2}$, Peter Storm ${ }^{3}$ and Kevin Whyte ${ }^{4}$
}

\begin{abstract}
We prove that there are only finitely many conjugacy classes of arithmetic maximal hyperbolic reflection groups.
\end{abstract}

Mathematics Subject Classification (2000). 20F55, 22E40, 11F06, $11 \mathrm{H} 56$.

Keywords. Hyperbolic, arithmetic, reflection, group.

\section{Introduction}

A hyperbolic reflection group $\Gamma$ is a discrete group generated by reflections in the faces of a hyperbolic polyhedron $P \subset \mathbb{H}^{n}$. We may assume that the dihedral angles of $P$ are of the form $\pi / n, n \geq 2$, in which case $P$ forms a fundamental domain for the action of $\Gamma$ on $\mathbb{H}^{n}$. If $P$ has finite volume, then $\mathbb{H}^{n} / \Gamma=\mathcal{O}$ is a hyperbolic orbifold of finite volume, which is obtained by "mirroring" the faces of $P$. A reflection group $\Gamma$ is maximal if there is no reflection group $\Gamma^{\prime}$ such that $\Gamma<\Gamma^{\prime}$. We defer the definition of arithmetic groups until later, but a theorem of Margulis implies that $\Gamma$ is arithmetic if and only if $[\operatorname{Comm}(\Gamma): \Gamma]=\infty$. Here we denote $\operatorname{Comm}(\Gamma)=$ $\left\{g \in \operatorname{Isom}\left(\mathbb{H}^{n}\right) \mid\left[\Gamma: g^{-1} \Gamma g \cap \Gamma\right]<\infty\right\}$ [23]. The main theorem of this article is that up to conjugacy in $\operatorname{Isom}\left(\mathbb{H}^{n}\right)$ there are only finitely many arithmetic groups which are maximal hyperbolic reflection groups. If one does not assume that the groups are maximal, then there is no corresponding finiteness result. For example, using a method of [33] one can see that there are right-angle reflection groups which contain infinitely many finite index right-angle reflection subgroups by sequentially doubling right-angled polyhedral fundamental domains along faces. Right-angled reflection groups are only known up to dimension 8. More recently, examples of

\footnotetext{
${ }^{1}$ Agol partially supported by NSF grant DMS-0504975 and the Guggenheim Foundation.

${ }^{2}$ Belolipetsky thanks MPIM in Bonn for hospitality and support during the summer of 2006.

${ }^{3}$ Storm partially supported by NSF grant DMS-0603711 and Kevin Whyte's NSF career grant DMS0349290.

${ }^{4}$ Whyte partially funded by NSF Career grant DMS-0349290 and the Sloan Foundation.
} 
infinite families of arithmetic reflection groups up to dimension 19 have been given by Allcock [2] using a similar doubling method.

Finiteness results of this sort were proven before by Nikulin [24], [25], [26] for dimension $n \geq 10$. Subsequently Vinberg showed that there are no uniform (i.e., cocompact) reflection groups in dimension $n \geq 30$ [40]. Our results are independent of their work in the sense that we obtain a finiteness result in each dimension and then appeal to the result of Prokhorov that reflection groups do not exist in high enough dimensions [30]. The argument of this paper generalizes an argument of LongMaclachlan-Reid [21], which implies that there are only finitely many arithmetic minimal (or congruence) hyperbolic 2-orbifolds with bounded genus. Their argument is in fact a generalization of an argument of Zograf [43], who reproved that there are only finitely many congruence groups $\Gamma$ commensurable with $\operatorname{PSL}(2, \mathbb{Z})$ such that $\mathbb{H}^{2} / \Gamma$ has genus 0 (This was proven originally by Dennin [9], [10], and was known as Rademacher's conjecture). These arguments were generalized in [1] to show that there are only finitely many maximal arithmetic reflection groups in dimension 3 . Thus, our result is only new for cocompact maximal arithmetic groups in dimensions $4 \leq n \leq 9$. One key ingredient of these arguments is a theorem of Burger and Sarnak [8], which implies that there is a lower bound on the first eigenvalue of a congruence arithmetic hyperbolic group defined by a quadratic form. The other key ingredient is an inequality of Li-Yau [20], generalized to orbifolds, which allows us to give an upper bound on the first eigenvalue of a maximal quotient of an arithmetic reflection group. One of the new ingredients of the present work is the use of some results on the distribution of covolumes of maximal arithmetic subgroups obtained in [3].

Remark. As this manuscript was being prepared, a short preprint appeared by Nikulin proving the finiteness of arithmetic maximal reflection groups in all dimensions [27]. Nikulin's proof makes use of Nikulin's previous results [24], [25], as well as the finiteness results for maximal arithmetic reflection groups in dimensions two [21] and three [1]. The methods in this paper are completely independent of Nikulin's methods. A final classification of maximal arithmetic reflection groups might combine the techniques of both approaches, so it seemed interesting to publish our result, even with the appearance of Nikulin's preprint.

\section{Conformal volume of orbifolds}

Conformal volume was first defined by Li-Yau [20], partially motivated by generalizing results on surfaces due to Yang-Yau [42], Hersch [15], and Szegô [35]. We generalize this notion to orbifolds. Let $(\mathcal{O}, g)$ be a complete Riemannian orbifold, possibly with boundary. Let $|\mathcal{O}|$ denote the underlying topological space. Denote the volume form by $d v_{g}$, and the volume by $\operatorname{Vol}(\mathcal{O}, g)$. Let $\operatorname{Möb}\left(\mathbb{S}^{n}\right)$ denote the conformal transformations of $\mathbb{S}^{n}$. It is well known that $\operatorname{Möb}\left(\mathbb{S}^{n}\right)=\operatorname{Isom}\left(\mathbb{H}^{n+1}\right)$. The 
topological space $|\mathcal{O}|$ has a dense open subset which is a Riemannian manifold. We will say that a map $\varphi:\left|\mathcal{O}_{1}\right| \rightarrow\left|\mathcal{O}_{2}\right|$ is $P C$ if it is a continuous map which is piecewise a conformal immersion. Clearly, if $\varphi:|\mathcal{O}| \rightarrow \mathbb{S}^{n}$ is $\mathrm{PC}$, and $\mu \in \operatorname{Möb}\left(\mathbb{S}^{n}\right)$, then $\mu \circ \varphi$ is also PC. Let $\left(\mathbb{S}^{n}\right.$, can) be the canonical round metric on the $n$-sphere.

Definition 2.1. For a piecewise smooth map $\varphi:|\mathcal{O}| \rightarrow\left(\mathbb{S}^{n}\right.$, can $)$, define

$$
V_{\mathrm{PC}}(n, \varphi)=\sup _{\mu \in \operatorname{Möb}\left(\mathbb{S}^{n}\right)} \operatorname{Vol}\left(\mathcal{O},(\mu \circ \varphi)^{*}(\mathrm{can})\right) .
$$

If there exists a PC map $\varphi:|\mathcal{O}| \rightarrow \mathbb{S}^{n}$, then we also define

$$
V_{\mathrm{PC}}(n, \mathcal{O})=\inf _{\varphi:|\mathcal{O}| \rightarrow \mathbb{S}^{n} \mathrm{PC}} V_{\mathrm{PC}}(n, \varphi) .
$$

Here $V_{\mathrm{PC}}(n, \mathcal{O})$ denotes the (n-dimensional) piecewise conformal volume of $\mathcal{O}$.

Remark. It seems likely that our definition of piecewise conformal volume coincides with conformal volume of $\mathrm{Li}$-Yau for manifolds, but we have not checked this (it would suffice to show that a PC map can be approximated by conformal maps).

If there exists a piecewise isometric map $\varphi:(|\mathcal{O}|, g) \rightarrow \mathbb{E}^{n}$ for some $n$, then clearly $V_{\mathrm{PC}}(n, \mathcal{O})$ is well defined, since $\mathbb{E}^{n}$ has a conformal embedding into $\mathbb{S}^{n}$. Let $\Sigma \subset \mathcal{O}$ be the singular locus of $\mathcal{O}$. By the Nash embedding theorem [14], there is an isometry $\rho: \mathcal{O} \backslash \Sigma \rightarrow \mathbb{E}^{N}$, for $N$ large enough. Then $\rho$ extends continuously to an isometric map $\bar{\rho}: \mathcal{O} \rightarrow \mathbb{E}^{N}$. Thus, $V_{\mathrm{PC}}(N, \mathcal{O})$ is well defined. We record some basic facts about conformal volume, generalizing some of the facts in Section 1 of [20] which carry over to our notion of conformal volume for orbifolds.

Fact 1: If $\mathcal{O}$ admits a PC map $\psi$ of geometric degree $d$ onto another orbifold $\mathcal{P}$, then

$$
V_{\mathrm{PC}}(n, \mathcal{O}) \leq|d| V_{\mathrm{PC}}(n, \mathcal{P}) .
$$

This follows because for any PC map $\varphi: \mathcal{P} \rightarrow \mathbb{S}^{n}$, the composition $\varphi \circ \psi: \mathcal{O} \rightarrow \mathbb{S}^{n}$ satisfies $V_{\mathrm{PC}}(n, \varphi \circ \psi) \leq d V_{\mathrm{PC}}(n, \varphi)$.

Fact 2: Since the embedding $\mathbb{S}^{n} \hookrightarrow \mathbb{S}^{n+1}$ is an isometry, it is clear that $V_{\mathrm{PC}}(n, \mathcal{O}) \geq$ $V_{\mathrm{PC}}(n+1, \mathcal{O})$. Define the piecewise conformal volume

$$
V_{\mathrm{PC}}(\mathcal{O})=\lim _{n \rightarrow \infty} V_{\mathrm{PC}}(n, \mathcal{O}) .
$$

Fact 3: If $\mathcal{O}$ is of dimension $m$, and $\varphi:|\mathcal{O}| \rightarrow \mathbb{S}^{n}$ is a PC map, then

$$
V_{\mathrm{PC}}(n, \varphi) \geq V_{\mathrm{PC}}\left(n, \mathbb{S}^{m}\right)=\operatorname{Vol}\left(\mathbb{S}^{m}\right) .
$$

The same argument as in Li-Yau works here: "blow up" about a smooth manifold point of $\varphi(|\mathcal{O}|)$ so that the image converges in the Hausdorff topology to a geodesic 
sphere of dimension $m$. More precisely, let $x \in \mathcal{O}$ be a manifold point of $\mathcal{O}$ such that the differential $d \varphi_{x}$ has rank $m$. Let $\mu_{t}: \mathbb{R}^{n+1} \rightarrow \mathbb{R}^{n+1}$ be a Möbius transformation preserving $\mathbb{S}^{n}$, fixing $\pm \varphi(x)$, and translating the origin $0 \in \mathbb{R}^{n+1}$ to $-t \varphi(x)$, $0 \leq t<1$. Let $U \subset \mathcal{O}$ be a small neighborhood of $x$ such that $\operatorname{rk}\left(d \varphi_{y}\right)=m$ for all $y \in U$. Then $\mu_{t}(\varphi(U))$ converges to the geodesic sphere of dimension $m$ going through $\pm \varphi(x)$, and tangent to $d \varphi_{x}\left(T_{x} \mathcal{O}\right)$ as $t \rightarrow 1$. Thus, we see that $V_{\mathrm{PC}}(n, \varphi) \geq \operatorname{Vol}\left(\mathbb{S}^{m}\right)$.

Fact 4: If $\mathcal{O}$ is an embedded codimension-0 suborbifold of the orbifold $\mathcal{P}$ and $\varphi:|\mathcal{P}| \rightarrow \mathbb{S}^{n}$ is PC, then $V_{\mathrm{PC}}(n, \varphi) \geq V_{\mathrm{PC}}\left(n,\left.\varphi\right|_{|\mathcal{O}|}\right)$. Thus, $V_{\mathrm{PC}}(n, \mathcal{P}) \geq V_{\mathrm{PC}}(n, \mathcal{O})$.

For example, suppose that $\iota: P \subset \mathbb{S}^{n}$ is an $n$-dimensional submanifold, with the conformal structure induced from the embedding. Then $V_{\mathrm{PC}}(n, \iota) \leq V_{\mathrm{PC}}\left(\mathbb{S}^{n}\right)$ by Fact 4 . But by Fact $3, V_{\mathrm{PC}}(n, \iota) \geq V_{\mathrm{PC}}\left(\mathbb{S}^{n}\right)$, and therefore we have shown that $V_{\mathrm{PC}}(n, \iota)=V_{\mathrm{PC}}(P)=V_{\mathrm{PC}}\left(\mathbb{S}^{n}\right)=\operatorname{Vol}\left(\mathbb{S}^{n}\right)$.

Fact 5: If $\mathcal{O}=\mathcal{O}_{1} \cup \mathcal{O}_{2}$ and $\varphi: \mathcal{O} \rightarrow \mathbb{S}^{n}$, then

$$
V_{\mathrm{PC}}(\mathcal{O}, \varphi) \leq V_{\mathrm{PC}}\left(\mathcal{O}_{1},\left.\varphi\right|_{\mathcal{O}_{1}}\right)+V_{\mathrm{PC}}\left(\mathcal{O}_{2},\left.\varphi\right|_{\mathcal{O}_{2}}\right)
$$

\section{Finite subgroups of $\mathrm{O}(n+1)$}

In this section we give a bound on the conformal volume of $\mathbb{S}^{n} / \Gamma$, where $\Gamma<\mathrm{O}(n+1)$ is a finite subgroup. For $\Gamma<\mathrm{O}(n+1)$ finite, define the orbifold $M_{\Gamma}^{n}=\mathbb{S}^{n} / \Gamma$.

Theorem 3.1. There is a function $C(n)$ such that if $\Gamma<\mathrm{O}(n+1)$, then

$$
V_{\mathrm{PC}}\left(M_{\Gamma}^{n}\right)<C(n)|\Gamma|^{2 n} .
$$

Proof. First, we need a lemma.

Lemma 3.2. If we have $k$ hyperplanes in $\mathbb{R}^{n}$ going through the origin, where $k>0$, $n>0$, then they separate $\mathbb{R}^{n}$ into at most $f(k, n)=2 \sum_{i=0}^{n-1}\left(\begin{array}{c}k-1 \\ i\end{array}\right)=O\left(k^{n-1}\right)$ regions.

Proof. We may assume that the hyperplanes are in general position. The proof is by lexicographic induction on $(k, n)$. Clearly, $f(k, 1)=2, f(1, n)=2$. Consider $k$ hyperplanes $\left\{P_{1}, \ldots, P_{k}\right\}, P_{i} \subset \mathbb{R}^{n}, k>1, n>1$. Then the hyperplanes $\left\{P_{1}, \ldots, P_{k-1}\right\}$ separate $\mathbb{R}^{n}$ into at most $f(k-1, n)$ regions by the inductive hypothesis. Also, $\left\{P_{1} \cap P_{k}, \ldots, P_{k-1} \cap P_{k}\right\}$ is a collection of $k-1$ hyperplanes in $P_{k} \cong \mathbb{R}^{n-1}$, and thus they divide $P_{k}$ into at most $f(k-1, n-1)$ regions by the inductive hypothesis. Each region of $\mathbb{R}^{n} \backslash\left(P_{1} \cup \cdots \cup P_{k-1}\right)$ which meets $P_{k}$ will be divided into two, and there are at most $f(k-1, n-1)$ such regions. The regions which do not meet $P_{k}$ will remain 
unchanged. Thus, $\left|\mathbb{R}^{n} \backslash\left(P_{1} \cup \cdots \cup P_{k}\right)\right| \leq f(k-1, n)+f(k-1, n-1)=f(k, n)$. This follows since

$$
\begin{aligned}
f(k-1, n)+f(k-1, n-1) & =2 \sum_{i=0}^{n-1}\left(\begin{array}{c}
k-2 \\
i
\end{array}\right)+2 \sum_{i=0}^{n-2}\left(\begin{array}{c}
k-2 \\
i
\end{array}\right) \\
& \left.=2 \sum_{i=0}^{n-1}\left[\begin{array}{c}
k-2 \\
i
\end{array}\right)+\left(\begin{array}{c}
k-2 \\
i-1
\end{array}\right)\right] \\
& =2 \sum_{i=0}^{n-1}\left(\begin{array}{c}
k-1 \\
i
\end{array}\right)=f(k, n) .
\end{aligned}
$$

We will actually find a piecewise isometric map $f: M_{\Gamma}^{n} \rightarrow \mathbb{S}^{N}$ for some $N$, in which the number of isometrically embedded pieces is bounded by a polynomial in $|\Gamma|=m$. This will immediately imply the result, using Facts $3-5$ about conformal volume.

Define the orbi-covering $\pi_{\Gamma}: \mathbb{S}^{n} \longrightarrow M_{\Gamma}^{n}$. Let $\Gamma=\left\{\gamma_{1}, \gamma_{2}, \ldots, \gamma_{m}\right\}$. For each element $\gamma \in \Gamma$, right multiplication by $\gamma$ induces a permutation of $\left\{\gamma_{1}, \gamma_{2}, \ldots, \gamma_{m}\right\}$, which we identify with a permutation of $\{1,2, \ldots, m\}$. Notice that this does not give an action of $\Gamma$, since composing right multiplications reverses the order of group multiplication. Use these permutations to define, for each $\gamma \in \Gamma$, a permutation of the coordinates of $\prod_{i=1}^{m} \overline{\mathbb{B}}^{n+1}$ given by

$$
\gamma \cdot\left(x_{1}, \ldots, x_{m}\right)=\left(x_{\gamma \cdot 1}, \ldots, x_{\gamma \cdot m}\right) .
$$

Define $N=m(n+1)-1$ and

$$
\Psi: \mathbb{S}^{n} \rightarrow \mathbb{S}^{N} \subset \prod_{i=1}^{m} \overline{\mathbb{B}}^{n+1}, \quad x \mapsto m^{-\frac{1}{2}}\left(\gamma_{1} x, \gamma_{2} x, \ldots, \gamma_{m} x\right) .
$$

Then $\mathbb{S}^{n}$ is taken by $\Psi$ to a totally geodesic submanifold of $\mathbb{S}^{N}$, since it is (up to rotation in $\mathrm{O}(N+1)$ ) the restriction of the diagonal map $\mathbb{R}^{n+1} \rightarrow \mathbb{R}^{N+1}$, rescaled to be an isometry. For each element $\gamma \in \Gamma$ the map $\Psi$ is $\gamma$-equivariant because

$$
\Psi(\gamma \cdot x)=m^{-\frac{1}{2}}\left(\gamma_{1} \cdot \gamma \cdot x, \ldots, \gamma_{m} \cdot \gamma \cdot x\right)=m^{-\frac{1}{2}}\left(\gamma_{\gamma \cdot 1} x, \ldots, \gamma_{\gamma \cdot m} x\right)=\gamma \cdot \Psi(x)
$$

for any $x \in \mathbb{S}^{n}$.

Let $\Sigma_{m}$ be the permutation group on $m$ elements. The map $\Psi$ therefore descends to a map

$$
\Upsilon: M_{\Gamma}^{n} \longrightarrow \mathbb{S}^{N} / \Sigma_{m}=Y .
$$

The action of $\Sigma_{m}$ on $\mathbb{S}^{N}$ induces an embedding $\Sigma_{m}<\mathrm{O}(N+1)$. By permuting the coordinates of $\mathbb{R}^{N+1}$, the symmetric group $\Sigma=\Sigma_{N+1}$ acts isometrically as a 
reflection group on $\mathbb{S}^{N}$ such that $\Sigma_{m}<\Sigma$. Similar to what we did above, define the orbi-covering $\pi_{\Sigma}: Y \longrightarrow M_{\Sigma}^{N}$ and an isometric embedding $\iota_{\Sigma}:\left|M_{\Sigma}^{N}\right| \longrightarrow \mathbb{S}^{N}$, which exists since we may identify $\left|M_{\Sigma}^{N}\right|$ with the Coxeter polyhedron for $\Sigma$. Let $\left\{S_{k}\right\}_{k \in K}$ be the collection of $\left(\begin{array}{c}N+1 \\ 2\end{array}\right)$ hyperspheres given by the fixed point sets of the reflections in $\Sigma$.

The goal is now to estimate $V_{\mathrm{PC}}\left(M_{\Gamma}^{n},\left(\iota_{\Sigma} \circ \pi_{\Sigma} \circ \Upsilon\right)\right)$ from above.

Consider how $\Psi\left(\mathbb{S}^{n}\right)$ is cut up by the collection $\left\{S_{k}\right\}_{k \in K}$. It is cut up by the planes $\left\{\Psi\left(\mathbb{S}^{n}\right) \cap S_{k}\right\}_{k \in K}$ into at most $f\left(\left(\begin{array}{c}N+1 \\ 2\end{array}\right), n+1\right)$ convex sub-polyhedra $\left\{C_{\sigma}, \sigma \in \Sigma\right\}$. (For most $\sigma \in \Sigma, C_{\sigma}=\emptyset$.) Then each $C_{\sigma}$ is isometrically and totally geodesically projected to $\mathbb{S}^{N}$ via the map $\iota_{\Sigma} \circ \pi_{\Sigma} \circ \Upsilon$. Postcomposition by a conformal automorphism of $\mathbb{S}^{N}$ can then make the volume of the image of $C_{\sigma}$ no more than the volume of a unit $n$-sphere. By the combination of facts 3 through 5 of Section 2, we can conclude that

$$
V_{\mathrm{PC}}\left(M_{\Gamma}^{n},\left(\iota_{\Sigma} \circ \pi_{\Sigma} \circ \Upsilon\right)\right) \leq f\left(\left(\begin{array}{c}
m(n+1) \\
2
\end{array}\right), n+1\right) \cdot \operatorname{Vol}\left(\mathbb{S}^{n}\right) \leq C(n)|\Gamma|^{2 n}
$$

for some constant $C(n)$.

Remark. It turns out that a finite subgroup $\Gamma<\mathrm{O}(n+1)$ does not necessarily embed in a finite reflection subgroup of $\mathrm{O}(n+1)$. Thus, the strategy of [1] does not generalize to higher dimensions. It is an interesting problem to try to get better estimates on $V_{\mathrm{PC}}\left(M_{\Gamma}^{n}\right)$. The method we have used to estimate this quantity is probably far from optimal. In fact, in the above theorem we have constructed maps which potentially have very high multiplicity, which is exactly the sort of thing one wants to avoid in estimating conformal volume.

\section{Congruence arithmetic hyperbolic orbifolds}

This section collects some properties of arithmetic hyperbolic orbifolds which will be needed in the next section. Given a number field $k$, let $\mathcal{O}$ denote its ring of integers, $\mathscr{D}_{k}$ the absolute value of the discriminant of $k$ and $\operatorname{deg}(k)=[k: \mathbb{Q}]$.

The group $H=\operatorname{Isom}\left(\mathbb{H}^{n}\right)$ can be identified with $\mathrm{O}_{0}(n, 1)$ - the subgroup of the orthogonal group $\mathrm{O}(n, 1)$ which preserves the upper-half space. We now define arithmetic and congruence subgroups of $H$.

Definition 4.1. Let $k$ be a totally real number field, $f$ a quadratic form of signature $(n, 1)$ defined over $k$ such that for every non-identity embedding $\sigma: k \rightarrow \mathbb{R}$ the form $f^{\sigma}$ is positive definite. Then the group $\Gamma=\mathrm{O}_{0}(f, \mathcal{O})$ of the integral automorphisms of $f$ is a discrete subgroup of $H$ via the inertia theorem. Such groups $\Gamma$ and subgroups of $H$ which are commensurable to them are called arithmetic subgroups of the simplest type. The field $k$ is called a field of definition of $\Gamma$ (and 
subgroups commensurable to $\Gamma$ in $H$ ). An arithmetic subgroup $\Gamma$ is called a congruence subgroup if there is a nonzero ideal $a \subset \mathcal{O}$ such that $\Gamma \supset \mathrm{O}_{0}(f, a)$, where $\mathrm{O}_{0}(f, \mathfrak{a})=\left\{g \in \mathrm{O}_{0}(f, \mathcal{O}) \mid g \equiv \mathrm{Id}(\bmod \mathfrak{a})\right\}$, the principal congruence subgroup of $\mathrm{O}_{0}(f, \mathcal{O})$ of level $a$ (see [29] for more on arithmetic groups). We will also apply this terminology to the corresponding quotient orbifolds.

In general, arithmetic subgroups of semisimple Lie groups are defined using algebraic groups. This way the classification of semisimple algebraic groups [37] implies a classification of possible types of arithmetic subgroups. It follows that for hyperbolic spaces of even dimension all arithmetically defined subgroups are arithmetic subgroups of the simplest type. For odd $n$ there is another family of arithmetic subgroups given as the groups of units of appropriate Hermitian forms over quaternion algebras. Moreover, if $n=7$ there is also the third type of arithmetic subgroups of $H$ which are associated to the Cayley algebra. The following result due to Vinberg shows that for our purpose it will be always sufficient to consider only arithmetic subgroups of the simplest type:

Lemma 4.2 ([39], Lemma 7). If $\Gamma$ contains an arithmetic subgroup generated by reflections then it is defined by a quadratic form.

The following lemma is a special case of Proposition 3.3 in [3] which was proved as an application and extension of the method of Borel-Prasad [5].

Lemma 4.3. Given $n>3$, there exist positive constants $c_{n}$ and $\delta_{n}$ such that if a maximal arithmetic subgroup $\Gamma<\operatorname{Isom}\left(\mathbb{H}^{n}\right)$ is defined over the field $k$, then $\operatorname{Vol}\left(\mathbb{H}^{n} / \Gamma\right) \geq c_{n} D_{k}^{\delta_{n}}$.

Proof. It was shown in [3], Section 3.3, that for almost all admissible fields $k$, if $\Gamma$ is an arithmetic subgroup of $H\left(=\operatorname{Isom}\left(\mathbb{H}^{n}\right)\right)$ defined over $k$, then

$$
\mu(H / \Gamma) \geq \frac{1}{2} \mathscr{D}_{k}^{\delta_{n}},
$$

where $\mu$ is a Haar measure on $H$ normalized in a certain way and $\delta_{n}$ is a positive constant which depends only on $n$ and can be computed explicitly. There is a constant $c=c(n)$ such that $\operatorname{Vol}\left(\mathbb{H}^{n} / \Gamma\right)=c \mu(H / \Gamma)$ (in fact, in our case $c$ is equal to the volume of the unit sphere in $\left.\mathbb{R}^{n}\right)$. Thus for all but finitely many $k, \operatorname{Vol}\left(\mathbb{H}^{n} / \Gamma\right) \geq$ $\frac{1}{2} c D_{k}^{\delta_{n}}$. We can extend this inequality to the remaining finite collection of fields by decreasing if necessary the constant $c$.

The next fact which we recall here may have been known for a long time. More recently, related questions in a different context were thoroughly studied by I. Horozov in his PhD thesis [16], [17]. Still we were not able to find a proof of the statement in the literature, so we will present an argument. 
Lemma 4.4. There exists a constant $e_{n}$ such that if an arithmetic subgroup $\Gamma<\operatorname{Isom}\left(\mathbb{H}^{n}\right)$ is defined by a quadratic form defined over $k$ and $C<\Gamma$ is a finite cyclic subgroup, then $|C| \leq e_{n} \operatorname{deg}(k)^{2 n+2}$.

Proof. Let $\Gamma$ be a subgroup of $H$ commensurable with the group of integral points of $G(k)=\mathrm{O}_{0}(f, k)$. It is known that if the center of $H$ is trivial (which is indeed the case for $\left.H=\operatorname{Isom}\left(\mathbb{H}^{n}\right)\right)$ then $\Gamma$ is contained in $G(k)$ [32], Proposition 1.2. We come to a question about the torsion in $G(k) \subset \mathrm{O}_{0}(f, k) \subset \mathrm{GL}(n+1, k)$.

Let $A \in \mathrm{GL}(m, k), m=n+1$ be a torsion element of order $t \geq 2$. Let $\lambda_{1}, \ldots, \lambda_{m}$ be the eigenvalues of $A$. As $A$ is a matrix over $k$, its eigenvalues split into groups of conjugates under the action of $\operatorname{Gal}(\bar{k} / k)$. Now $A^{t}=\mathrm{Id}$ implies that the eigenvalues are roots of unity. Let $t_{1}, \ldots, t_{l}$ be their orders, so $t=\operatorname{lcm}\left(t_{1}, \ldots, t_{l}\right)$. If $\lambda$ is an eigenvalue then all its $\operatorname{Gal}(\bar{k} / k)$-conjugates are also eigenvalues of $A$, which implies

$$
\phi_{k}\left(t_{1}\right)+\cdots+\phi_{k}\left(t_{l}\right) \leq m,
$$

where $\phi_{k}(t)$ denotes a generalized Euler $\phi$-function, which can be defined as the degree over $k$ of the cyclotomic extension $k\left(\mu_{t}\right)$, where $\mu_{t}$ denotes a primitive $t$-th root of unity.

It is clear that the following inequalities are satisfied for $\phi_{k}(t)$ and the Euler $\phi$-function:

$$
\phi(t) / \operatorname{deg}(k) \leq \phi_{k}(t) \leq \phi(t) .
$$

To simplify the notation let $\operatorname{deg}(k)=d$. We have

$$
\phi\left(t_{i}\right) \leq m d .
$$

This implies

$$
\phi(t) \leq \phi\left(t_{1}\right) \ldots \phi\left(t_{l}\right) \leq(m d)^{m}
$$

(the first inequality employs the fact that $t=1 \mathrm{~cm}\left(t_{1}, \ldots, t_{l}\right)$ ).

Now using the well-known inequality $\phi(t) \geq \sqrt{t} / 2$, we obtain that

$$
t \leq 4(m d)^{2 m} \leq e_{n} d^{2 n+2} .
$$

Corollary 4.5. Under the hypothesis of the above lemma, there exists a constant $m_{n}$ such that if $F<\Gamma$ is a finite subgroup, then $|F| \leq m_{n} \operatorname{deg}(k)^{n(n+1)}$.

Proof. By Margulis' lemma, there is a constant $M_{n}$ such that if $F<\mathrm{O}(n)$, then there is an abelian subgroup $A<F$ with $[F: A] \leq M_{n}$ (see e.g. [36], Corollary 4.1.11). We may find common complex eigenspaces of the elements of $A: U_{1}, \ldots, U_{k}$, and real eigenspaces $V_{1}, \ldots, V_{l}$, where $k / 2+l \leq n$, such that $A$ acts on $U_{i}$ as a cyclic group $A_{i}$, and $A$ acts on $V_{i}$ as \pm 1 . We may embed $A$ in $\prod_{i=1}^{k} A_{i} \times(\mathbb{Z} / 2 \mathbb{Z})^{l}$, acting on $\prod_{i=1}^{k} U_{1} \times \prod_{j=1}^{l} V_{j}$. Thus $|A| \leq 2^{l} \prod_{i=1}^{k}\left|A_{i}\right|$. By the previous lemma, $\left|A_{i}\right| \leq e_{n} \operatorname{deg}(k)^{2 n+2}$, since a generator of $A_{i}$ is the projection of an element of $A$. Thus, $|F| \leq M_{n}|A| \leq m_{n} \operatorname{deg}(k)^{n(n+1)}$. 
Lemma 4.6. There exists $c>1$ such that $\mathscr{D}_{k}>c^{\operatorname{deg}(k)}$ for a number field $k$.

This follows from Minkowski's theorem (see e.g. [19], V.4). However, if one wants to obtain a "good value" for the constant $c$, the question becomes much more complicated. There has been a considerable amount of research in this direction. We refer to [28] for a survey of the results available so far.

Lemma 4.7. Maximal arithmetic subgroups of the simplest type are congruence.

Proof. Let $\Gamma$ be a maximal arithmetic subgroup which is commensurable with $G(\mathcal{O})=\mathrm{O}_{0}(f, \mathcal{O})$ for some quadratic form $f$ defined over $k$. There exists an $\mathcal{O}$ lattice $L$ in $V=k^{n+1}$ such that $\Gamma \cap G(k)(=\Gamma)=G^{L}=\{g \in G(k) \mid g(L)=L\}$ (see [29], Proposition 4.2, for $k=\mathbb{Q}$, the general case is entirely similar as we do not claim that $L$ is a free $\mathcal{O}$-lattice). Let also $M$ be the standard lattice in $V$ so that $G^{M}=G(\mathcal{O})$. We claim that there exists a principal congruence subgroup of $G^{M}$ which is contained in $G^{L}$. The argument is the same as in [29], Lemma 4.1.1: Let $\phi: G \rightarrow G$ be a $k$-isomorphism defined by the change of basis of $V$ from the standard basis (of $M$ ) to a $k$-basis contained in $L$. It follows that if $\Delta$ is a principal congruence subgroup of $G^{M}$ with respect to an ideal $a \subset \mathcal{O}$ such that the coefficients of $a(\phi-$ Id $)$ in the standard basis are integral, then $\Delta \subset G^{L}$.

Remark. The results which were discussed in this section so far are valid for arbitrary arithmetic subgroups, the proofs for the general case are similar to the case of the simplest type which we considered in detail.

The following theorem was stated in the case of arithmetic groups defined over $\mathbb{Q}$, but it is true in general. It is a translation of results of Jacquet-Langlands [18] and Gelbart-Jacquet [13] in the theory of automorphic forms into a statement about eigenvalues of congruence arithmetic groups, and it generalizes a famous theorem of Selberg for congruence subgroups of $\operatorname{PSL}(2, \mathbb{Z})[34]$.

Theorem 4.8 (Vigneras [38]). Let $\mathcal{O}=\mathbb{H}^{2} / \Gamma$, where $\Gamma$ is an arithmetic congruence Fuchsian subgroup of $\operatorname{Isom}\left(\mathbb{H}^{2}\right)$. Then $\lambda_{1}(\mathcal{O}) \geq \frac{3}{16}$.

In the above theorem, it is conjectured that $\lambda_{1}(\mathcal{O}) \geq \frac{1}{4}$, which generalizes a conjecture of Selberg about congruence subgroups of $\operatorname{PSL}(2, \mathbb{Z})$. The following theorem of Burger and Sarnak extends this result to higher dimensions by inducing from the 2-dimensional case.

Theorem 4.9 ([8], Corollary 1.3(a)). Let $\mathcal{O}=\mathbb{H}^{n} / \Gamma$, where $\Gamma$ is an arithmetic congruence subgroup defined by a quadratic form, $n \geq 3$ (or if $n=3$, $\Gamma$ need only be arithmetic congruence). Then $\lambda_{1}(\mathcal{O}) \geq \frac{2 n-3}{4}$.

Under the hypotheses of Theorem 4.9, it is conjectured that $\lambda_{1}(\mathcal{O}) \geq n-2$, which is a special case of the generalized Ramanujan conjecture [8], Corollary 1.3 (b). 


\section{Finiteness of arithmetic hyperbolic maximal reflection groups}

We put together the results in the previous sections to prove our main theorem.

Theorem 5.1. There are only finitely many conjugacy classes of arithmetic maximal reflection groups in $\operatorname{Isom}\left(\mathbb{H}^{n}\right)$.

Proof. Suppose that $\Gamma$ is an arithmetic maximal reflection group. Then there is no group $\Gamma^{\prime}<\operatorname{Isom}\left(\mathbb{H}^{n}\right)$ such that $\Gamma<\Gamma^{\prime}$ and $\Gamma^{\prime}$ is generated by reflections. There does exist a maximal lattice $\Gamma_{0}<\operatorname{Isom}\left(\mathbb{H}^{n}\right)$ satisfying $\Gamma \leq \Gamma_{0}$. The group $\Gamma$ is generated by reflections in a finite volume polyhedron $P$, which forms a fundamental domain for $\Gamma$.

Lemma 5.2 (Vinberg [39]). $\Gamma$ is a normal subgroup of $\Gamma_{0}$. Moreover, there is a finite subgroup $\Theta<\Gamma_{0}$ such that $\Theta \rightarrow \Gamma_{0} / \Gamma$ is an isomorphism, and $\Theta$ is the group of symmetries of the polyhedron $P$.

Proof. This follows from the fact that the set of reflections in $\Gamma_{0}$ is conjugacy invariant, and therefore the subgroup of $\Gamma_{0}$ generated by reflections in $\Gamma_{0}$ is normal in $\Gamma_{0}$. Since $\Gamma$ is a maximal reflection group, this subgroup of $\Gamma_{0}$ must be $\Gamma$.

Suppose that there is an element $\gamma \in \Gamma_{0}$ such that $\operatorname{int}(P) \cap \gamma(\operatorname{int}(P)) \neq \emptyset$ and $\gamma(P) \neq P$. Then there is a geodesic plane $V$ containing a face of $P$ such that $\gamma(V) \cap \operatorname{int}(P) \neq \emptyset$. The reflection $r_{V} \in \Gamma$ in the plane $V$ is conjugate to a reflection $r_{\gamma(V)}=\gamma r_{V} \gamma^{-1}$, which is not in $\Gamma$ since $r_{\gamma(V)}(\operatorname{int}(P)) \cap \operatorname{int}(P) \neq \emptyset$. However $\Gamma \unlhd \Gamma_{0}$ implies $r_{\gamma(V)} \in \Gamma$, a contradiction.

Let $\Theta$ be the subgroup of $\Gamma_{0}$ such that $\Theta(P)=P$. Then $\Theta$ is finite because $P$ is finite volume and has finitely many faces.

Consider the composition $\Theta \hookrightarrow \Gamma_{0} \rightarrow \Gamma_{0} / \Gamma$. The group $\Gamma \cap \Theta$ is trivial because $P$ is a fundamental domain for $\Gamma$. The composition map is therefore injective. To see surjectivity, pick a $\gamma_{0} \in \Gamma_{0}$ and find a $\gamma \in \Gamma$ such that $\gamma_{0}^{-1}(\operatorname{int}(P)) \cap \gamma(\operatorname{int}(P)) \neq \varnothing$. Then $\gamma_{0} \gamma(P)=P$, so $\gamma_{0} \gamma=\theta \in \Theta$. Therefore $\gamma_{0} \Gamma=\theta \Gamma$. This shows that the composition $\Theta \rightarrow \Gamma_{0} / \Gamma$ is surjective and an isomorphism.

Let $\mathcal{O}=\mathbb{H}^{n} / \Gamma_{0}$ and let $\Theta$ be the finite group coming from the previous lemma.

Now we remark that from the result of Long-Maclachlan-Reid [21] the case of $n=2$ follows. If $n=2$ then there is a subgroup $\Gamma^{\prime}<\Gamma_{0}$ of index 2 which is a genus 0 Fuchsian group. Thus, by Theorem 1.1 of [21], we can conclude that there are only finitely many possible $\Gamma^{\prime}$, since $\Gamma^{\prime}$ is also a congruence group, and therefore there are only finitely many $\Gamma_{0}$ and $\Gamma$. In fact, Area $\left(\mathbb{H}^{2} / \Gamma^{\prime}\right)<\frac{128}{3} \pi$. Also, the case of $n=3$ was proven in [1]. So we may restrict to the case that $4 \leq n \leq 995$, by Prokhorov's theorem [30]. In fact, since Nikulin has shown the finiteness of maximal non-uniform arithmetic reflection groups [24], [25], [26], we may restrict to the case 
that $\Gamma$ is cocompact, and therefore $n<30$, although our argument gives a new proof of Nikulin's theorem, making use of Prokhorov's theorem.

Consider $\mathbb{H}^{n} \subset \mathbb{S}^{n}$ embedded conformally as the upper hemisphere of $\mathbb{S}^{n}$, so that Isom $\left(\mathbb{H}^{n}\right)$ acts conformally on $\mathbb{S}^{n}$. Normalize so that $\Theta$ acts isometrically on $\mathbb{S}^{n}$ by placing the common fixed point of $\Theta$ at the north pole of $\mathbb{S}^{n}$. Clearly, $V_{\mathrm{PC}}(n, \mathcal{O})=$ $V_{\mathrm{PC}}(n, P / \Theta)$ since $|\mathcal{O}|=|P / \Theta|$. Then the orbifold $P / \Theta \subset \mathbb{S}^{n} / \Theta$ is a conformal embedding, so Fact 4 of Section 2 gives the inequality $V_{\mathrm{PC}}(n, P / \Theta) \leq V_{\mathrm{PC}}\left(n, \mathbb{S}^{n} / \Theta\right)$.

It will follow from Theorem 6.2 proven in the next section that for $\mathcal{O}$ a hyperbolic $m$-orbifold,

$$
\lambda_{1}(\mathcal{O}) \operatorname{Vol}(\mathcal{O})^{\frac{2}{m}} \leq m V_{\mathrm{PC}}(m, \mathcal{O})^{\frac{2}{m}}
$$

We now apply the eigenvalue estimates from Theorems 6.2 and 4.9:

$$
\frac{2 n-3}{4} \operatorname{Vol}(\mathcal{O})^{\frac{2}{n}} \leq \lambda_{1}(\mathcal{O}) \operatorname{Vol}(\mathcal{O})^{\frac{2}{n}} \leq n V_{\mathrm{PC}}(n, \mathcal{O})^{\frac{2}{n}} \leq n\left(V_{\mathrm{PC}}\left(\mathbb{S}^{n} / \Theta\right)\right)^{\frac{2}{n}}
$$

Assume now that $\Gamma_{0}$ is defined over the number field $k$. By Theorem 3.1 and Corollary 4.5 we have

$$
V_{\mathrm{PC}}\left(\mathbb{S}^{n} / \Theta\right) \leq C(n)|\Theta|^{2 n} \leq C(n)\left(m_{n} \operatorname{deg}(k)^{2(n+1) n}\right)^{2 n} .
$$

By Lemmas 4.3 and 4.6, we have $\operatorname{Vol}(\mathcal{O})=\operatorname{Vol}\left(\mathbb{H}^{n} / \Gamma_{0}\right) \geq c_{n} c^{\delta_{n} \operatorname{deg}(k)}$. Putting these inequalities together, we obtain an inequality of the form

$$
c^{\delta_{n} \operatorname{deg}(k)} \leq C_{1}(n) \operatorname{deg}(k)^{4(n+1) n^{2}},
$$

which implies that $\operatorname{deg}(k)$ must be bounded.

We also obtain

$$
\operatorname{Vol}(\mathcal{O})^{\frac{2}{n}} \leq \frac{4 n}{2 n-3} C(n)^{\frac{2}{n}} m_{n}^{4} \operatorname{deg}(k)^{8(n+1) n}
$$

Since $\operatorname{deg}(k)$ is bounded, this gives an upper bound on $\operatorname{Vol}(\mathcal{O})$ depending only on $n$.

Since volumes of arithmetic hyperbolic orbifolds are discrete [4], [41], and $\Gamma$ is uniquely determined by $\Gamma_{0}$, we conclude that there are only finitely many arithmetic maximal reflection groups in dimension $n$.

\section{Eigenvalue bounds}

In this section we observe that the argument of [11], Theorem 2.2, generalizes to our context of orbifolds (their theorem sharpens Corollary 3 from Section 2 of [20]). If $(\mathcal{O}, g)$ is a Riemannian orbifold, then $\lambda_{1}(\mathcal{O}, g)$ is the first non-zero eigenvalue of the operator $-\widetilde{\Delta}_{g}$ on $\mathcal{O}$, where $\widetilde{\Delta}_{g}$ is the unique self-adjoint extension of the Laplacian on $\mathcal{O}$. We will be using the variational characterization of $\lambda_{1}(\mathcal{O}, g)$ in terms of the Rayleigh-Ritz quotient. 
Lemma 6.1. Let $\mathcal{O}$ be an orbifold of finite volume. Let $f \in W^{1,2}(\mathcal{O})$ be an $L^{2}$-function with $L^{2}$-distributional derivative and such that $\int_{\mathcal{O}} f d v_{g}=0$. Then

$$
\lambda_{1}(\mathcal{O}, g) \int_{\mathcal{O}} f^{2} d v_{g} \leq \int_{\mathcal{O}}|\nabla f|^{2} d v_{g}
$$

Proof. Let $A$ be a self-adjoint operator on a Hilbert space $\mathscr{H}$ such that $A$ is semibounded below. This means that there exists a constant $k$ such that $\langle\psi, A \psi\rangle \geq$ $k\langle\psi, \psi\rangle$ for all $\psi \in \mathscr{H}$. The Laplacian $\Delta$ with domain restricted to compactly supported, $C^{\infty}$ functions on $\mathcal{O}$ is essentially self-adjoint ([12], Theorem 1.9), with self-adjoint extension $\widetilde{\Delta}$. In the case of the self-adjoint operator $-\widetilde{\Delta}$, we have $-\widetilde{\Delta}$ is semi-bounded below, with constant $k=0$. This follows from the fact that $C_{c}^{\infty}(\mathcal{O})$ is dense in $\operatorname{Domain}(\widetilde{\Delta})$, and for $f \in C_{c}^{\infty}(\mathcal{O})$ integration by parts gives $\int_{\mathcal{O}}-f \Delta f d v_{g}=\int_{\mathcal{O}}|\nabla f|^{2} d v_{g}$, which is non-negative. We have $\lambda_{0}(-\widetilde{\Delta})=0$, with constant eigenfunction. Therefore, the Rayleigh-Ritz principle for semi-bounded below operators [31], XIII, gives

$$
\lambda_{1}(\mathcal{O}, g)=\inf \left\{\frac{\int_{\mathcal{O}}-f \tilde{\Delta} f d v_{g}}{\int_{\mathcal{O}} f^{2} d v_{g}} \mid f \in \operatorname{Domain}(\widetilde{\Delta})-0, \int_{\mathcal{O}} f d v_{g}=0\right\} .
$$

Since $C_{c}^{\infty}(\mathcal{O})$ is dense in $\operatorname{Domain}(\widetilde{\Delta})$, we may take the above infimum over functions $f \in C_{c}^{\infty}(\mathcal{O})$. Then integrating by parts, we have

$$
\lambda_{1}(\mathcal{O}, g)=\inf \left\{\frac{\int_{\mathcal{O}}|\nabla f|^{2} d v_{g}}{\int_{\mathcal{O}} f^{2} d v_{g}} \mid f \in C_{c}^{\infty}(\mathcal{O})-0, \int_{\mathcal{O}} f d v_{g}=0\right\} .
$$

Then we use again the fact that $C_{c}^{\infty}(\mathcal{O})$ is dense in the Sobolev space $W^{1,2}(\mathcal{O})$ to see that we may take the above infimum over functions in $W^{1,2}(\mathcal{O})$.

Theorem 6.2 (see [11], Theorem 2.2). Let $(\mathcal{O}, g)$ be a Riemannian orbifold of dimension $m$ of finite volume. If $\varphi:|\mathcal{O}| \rightarrow \mathbb{S}^{n}$ is a PC map, then

$$
\lambda_{1}(\mathcal{O}, g) \operatorname{Vol}(\mathcal{O}, g)^{\frac{2}{m}} \leq m V_{\mathrm{PC}}(n, \varphi)^{\frac{2}{m}} .
$$

Proof. Let $\boldsymbol{X}=\left(X_{1}, \ldots, X_{n+1}\right)$ be the coordinate functions on $\mathbb{R}^{n+1}$. Then $\sum_{i=1}^{n+1} X_{i}^{2}=1$, restricted to the unit sphere $\mathbb{S}^{n} \subset \mathbb{R}^{n+1}$.

Lemma 6.3. There exists $\mu \in \operatorname{Möb}\left(\mathbb{S}^{n}\right)$ such that $\int_{\mathcal{O}} \boldsymbol{X} \circ \mu \circ \varphi d v_{g}=\mathbf{0}$.

Proof. Let $\boldsymbol{x} \in \mathbb{S}^{n}$. For $0 \leq t<1, t \boldsymbol{x} \in \mathbb{B}^{n+1}$ (the open unit ball), let $\mu_{t \boldsymbol{x}} \in$ $\operatorname{Möb}\left(\mathbb{S}^{n}\right)=\operatorname{Isom}\left(\mathbb{B}^{n+1}\right)$ be the hyperbolic translation along the ray $\mathbb{R} \boldsymbol{x}$ taking $\mathbf{0}$ to $t \boldsymbol{x}$ (thus, $\mu_{0 \boldsymbol{x}}=\mu_{\mathbf{0}}=\mathrm{Id}$ ). Let $H(t \boldsymbol{x})=\frac{1}{\operatorname{Vol}(\mathcal{O}, g)} \int_{\mathcal{O}} \boldsymbol{X} \circ \mu_{t \boldsymbol{x}} \circ \varphi d v_{g}$. We may think of $H$ as defining a continuous function $H: \mathbb{B}^{n+1} \rightarrow \mathbb{B}^{n+1}$, which gives the center of mass of the measure coming from $\varphi_{*} d v_{g}$, where we take the point $-t \boldsymbol{x}$ to the origin 
of the sphere $\mathbb{S}^{n}=\partial \mathbb{B}^{n+1}$ by the conformal map $\mu_{t \boldsymbol{x}}$. Let $x_{i} \in \mathbb{B}^{n+1}$ be a sequence of points approaching $x_{\infty} \in \mathbb{S}^{n}$. We want to show that $H$ extends to a map fixing $\partial \mathbb{B}^{n+1}$. To see this, let $U_{\delta}=\mathbb{S}^{n} \backslash B_{\delta}\left(-x_{\infty}\right)$ be a neighborhood of $x_{\infty}$. Then the fact that $\varphi$ is PC implies that for any $\varepsilon>0$, there exists $\delta>0$ such that $\operatorname{Vol}\left(\varphi^{-1}\left(U_{\delta}\right)\right)>$ $\operatorname{Vol}(\mathcal{O})(1-\varepsilon)$. Then we may find $N>0$ such that $\mu_{x_{i}}\left(U_{\delta}\right) \subset B_{\varepsilon}\left(x_{\infty}\right)$ for $i>N$. Thus, we may see that $H\left(x_{i}\right) \in B_{\varepsilon}\left(x_{\infty}\right)$. So $\lim _{i \rightarrow \infty} H\left(x_{i}\right)=x_{\infty}$. So we see that $H$ extends to a continuous function $\bar{H}: \overline{\mathbb{B}}^{n+1} \rightarrow \overline{\mathbb{B}}^{n+1}$ such that $\left.\bar{H}\right|_{\mathbb{S}^{n}}=\left.\mathrm{Id}\right|_{\mathbb{S}^{n}}$, and $\left.\bar{H}\right|_{\mathbb{B}^{n+1}}=H$. Thus, $H$ is onto, so there exists $\mathbf{y} \in \mathbb{B}^{n+1}$ such that $H(\mathbf{y})=\mathbf{0}$, and we take $\mu=\mu_{\mathbf{y}}$.

Now replace $\varphi$ with $\mu \circ \varphi$, noting that this is still a PC map. Then the $X_{i} \circ \varphi$ may be used as test functions in the Rayleigh quotient, since they are $L^{2}$-orthogonal to the constant function and lie in the Sobolev space $W^{1,2}(\mathcal{O})$ because they are piecewise smooth (they are differentiable a.e., with (distributional) derivative lying in $L^{2}(\mathcal{O})$; this follows from equations (2) and (3) below if $\operatorname{Vol}\left(\mathcal{O}, \varphi^{*}\right.$ can) $<\infty$, which we may assume when $\left.V_{\mathrm{PC}}\left(\mathcal{O}, \mathbb{S}^{n}\right)<\infty\right)$. Thus,

$$
\lambda_{1}(\mathcal{O}) \int_{\mathcal{O}}\left|X_{i} \circ \varphi\right|^{2} d v_{g} \leq \int_{\mathcal{O}}\left|\nabla X_{i} \circ \varphi\right|^{2} d v_{g}
$$

Summarizing, we see that

$$
\begin{aligned}
\lambda_{1}(\mathcal{O}) \operatorname{Vol}(\mathcal{O}, g) & =\lambda_{1}(\mathcal{O}) \int_{\mathcal{O}} \sum_{i=1}^{n+1}\left|X_{i} \circ \varphi\right|^{2} d v_{g} \\
& \leq m \int_{\mathcal{O}} \frac{1}{m} \sum_{i=1}^{n+1}\left|\nabla X_{i} \circ \varphi\right|^{2} d v_{g} \\
& \leq m\left(\int_{\mathcal{O}}\left(\frac{1}{m} \sum_{i=1}^{n+1}\left|\nabla X_{i} \circ \varphi\right|^{2}\right)^{\frac{m}{2}} d v_{g}\right)^{\frac{2}{m}} \operatorname{Vol}(\mathcal{O}, g)^{1-\frac{2}{m}}
\end{aligned}
$$

where the last inequality is Hölder's inequality.

Take a point $x \in \mathcal{O}$ at which $\varphi$ is differentiable and conformal (by the definition of a PC map, almost every point of $\mathcal{O}$ satisfies these two conditions). After possibly performing an orthogonal change of coordinates at $x \in \mathcal{O}$ and on $\mathbb{R}^{n+1}$, we may assume without loss of generality that the derivative $d \varphi(x): T_{x}(\mathcal{O}) \rightarrow T_{\varphi(x)}\left(\mathbb{R}^{n+1}\right)$ may be written as

$$
d \varphi=\left[\begin{array}{ccc}
k & 0 & 0 \\
0 & \ddots & 0 \\
0 & 0 & k \\
0 & 0 & 0 \\
0 & 0 & 0
\end{array}\right],
$$


where $k$ is the dilatation factor of $\varphi$ at $x$. From the fact that $\operatorname{tr}\left(A^{t} A\right)$ is invariant under pre- or post-composition with an orthogonal matrix, one sees that

$$
\frac{1}{m} \sum_{i=1}^{n+1}\left|\nabla X_{i} \circ \varphi\right|^{2}=\frac{1}{m} \operatorname{tr}\left(d \varphi(x)^{t} d \varphi(x)\right)=k^{2} .
$$

Since $\varphi^{*}(\operatorname{can})(x)=k^{m} d v_{g}(x)$, where can is the volume form on $\mathbb{S}^{n}$, we see that

$$
\left(\frac{1}{m} \sum_{i=1}^{n+1}\left|\nabla X_{i} \circ \varphi\right|^{2}\right)^{m / 2} d v_{g}=\varphi^{*}(\text { can }) \text { a.e. }
$$

This yields the inequality

$$
\int_{\mathcal{O}}\left(\frac{1}{m} \sum_{i=1}^{n+1}\left|\nabla X_{i} \circ \varphi\right|^{2}\right)^{\frac{m}{2}} d v_{g} \leq \operatorname{Vol}\left(\mathcal{O}, \varphi^{*} \text { can }\right) .
$$

Finally, we obtain the desired inequality by combining inequalities (2) and (3), and taking an infimum over all PC functions $\varphi$.

\section{Conclusion}

It is clear that for a finite volume polyhedron $P \subset \mathbb{H}^{n}, V_{\mathrm{PC}}(n, P)=\operatorname{Vol}\left(\mathbb{S}^{n}\right)$, so, by Theorem 6.2,

$$
\lambda_{1}(P) \operatorname{Vol}(P)^{\frac{2}{n}} \leq n \operatorname{Vol}\left(\mathbb{S}^{n}\right)^{\frac{2}{n}} .
$$

Thus in $n$ dimensions there are finitely many reflection groups $\Gamma<\operatorname{Isom}\left(\mathbb{H}^{n}\right)$ which have a lower bound on $\lambda_{1}\left(\mathbb{H}^{n} / \Gamma\right)$. If it were true that maximal arithmetic hyperbolic reflection groups are congruence, then this would simplify the argument in this paper quite a bit, since we would immediately get an upper bound on the volume by the above observation. So it is natural to ask: does there exist a non-congruence maximal arithmetic reflection group?

The following conjecture seems to be interesting and would also give an alternative argument to the main theorem of this paper:

Conjecture. There is a function $K(n)$ such that if $\mathcal{O}$ is an elliptic $n$-orbifold, then $V_{\mathrm{PC}}(\mathcal{O}) \leq K(n)$.

Another approach to the proof of the main theorem would be possible if we assume the validity of the short geodesic conjecture for arithmetic hyperbolic $n$-orbifolds. As was pointed out earlier in this section, Theorem 6.2 implies that

$$
\lambda_{1}(P) \operatorname{Vol}(P)^{\frac{2}{n}} \leq n \operatorname{Vol}\left(\mathbb{S}^{n}\right)^{\frac{2}{n}} .
$$


Now if the group $\Gamma$ generated by reflections in $P$ is arithmetic, then it is contained in some maximal arithmetic subgroup $\Gamma_{0}<\operatorname{Isom}\left(\mathbb{H}^{n}\right)$ with a finite index $l$. Let $k$ be the field of definition of $\Gamma_{0}$. Corollary 4.5 and Lemma 5.2 imply that $l \leq$ $m_{n} \operatorname{deg}(k)^{2(n+1) n}$, and from Lemmas 4.3 and 4.6 it follows that $\operatorname{Vol}(P) \geq l c^{\delta_{n} \operatorname{deg}(k)}$. What remains is to estimate $\lambda_{1}(P)=\lambda_{1}(\Gamma)$ using the fact that $\lambda_{1}\left(\Gamma_{0}\right) \geq(2 n-3) / 4$, which follows from Theorem 4.9. This brings us to the question about the spectrum of coverings which was studied by Brooks in [6], [7]. The result of [7] implies that $\lambda_{1}(\Gamma) \geq c\left(\Gamma_{0}\right) \lambda_{1}\left(\Gamma_{0}\right) / l^{2}$, and more careful analysis of the proof shows that the constant $c\left(\Gamma_{0}\right)$ depends only on the lower bound for the injectivity radius of $\mathbb{H}^{n} / \Gamma_{0}$. The short geodesic conjecture says that in every dimension $n \geq 3$ there exist a universal lower bound for the injectivity radius, so assuming the conjecture is true we can write $\lambda_{1}(\Gamma) \geq c(n) \lambda_{1}\left(\Gamma_{0}\right) / l^{2}$. Substituting this into the main inequality together with the bounds for the index $l$ and $\operatorname{Vol}(P)$, we obtain the finiteness results in a similar way to the proof of the theorem in Section 5. This argument avoids the analysis of the conformal volumes of spherical orbifolds (Section 3 ) but unfortunately is based on an open conjecture.

From the main theorem we conclude that given an arithmetic reflection group $\Gamma<\operatorname{Isom}\left(\mathbb{H}^{n}\right)$, it must lie in one of finitely many maximal reflection groups (up to conjugacy). If $\Gamma$ is a reflection group in a polyhedron $P$ for which all the dihedral angles are $\pi / 2$ or all are $\pi / 3$, then there are infinitely many finite covolume reflection subgroups of $\Gamma$. Thus we see that there are commensurability classes of arithmetic groups for which there are infinitely many reflection groups in the commensurability class, and thus in our finiteness result, the maximality assumption is crucial. The same is true for the arithmeticity assumption. For example, consider the groups generated by reflections of the hyperbolic plane in the sides of a hyperbolic triangle with angles $\pi / 2, \pi / 3, \pi / m(m \geq 7)$. These groups are known to be maximal discrete subgroups of $\operatorname{Isom}\left(\mathbb{H}^{2}\right)$ but all except finitely many of them are non-arithmetic. A similar construction is available for hyperbolic 3 -space with triangular prisms replacing triangles [22].

It is an interesting project to try to identify all arithmetic maximal reflection groups and to classify their reflection subgroups of finite covolume. Although all the constants in our finiteness argument are computable, the quantitative estimates which could be deduced directly from the proof are unreasonably large. However, if we restrict to the congruence reflection groups (see the discussion at the beginning of this section) or make some additional assumptions on the groups of automorphisms of the fundamental polyhedra of the reflection groups, then the bounds can be improved essentially.

\section{References}

[1] I. Agol, Finiteness of arithmetic Kleinian reflection groups. In Proc. Internat. Congr. Mathematicians (Madrid 2006). Vol. II, EMS Publishing House, Zürich 2006, 951-960. Zbl 1102.30042 MR 2275630 
[2] D. Allcock, Infinitely many hyperbolic Coxeter groups through dimension 19. Geom. Topol. 10 (2006), 737-758. Zbl 1133.20025 MR 2240904

[3] M. Belolipetsky, Counting maximal arithmetic subgroups. Duke Math. J. 140 (2007), 1-33. Zbl 1131.22008 MR 2355066

[4] A. Borel, Commensurability classes and volumes of hyperbolic 3-manifolds. Ann. Scuola Norm. Sup. Pisa Cl. Sci. (4) 8 (1981), 1-33. Zbl 0473.57003 MR 616899

[5] A. Borel and G. Prasad, Finiteness theorems for discrete subgroups of bounded covolume in semi-simple groups. Inst. Hautes Études Sci. Publ. Math. 69 (1989), 119-171; addendum ibid. 71 (1990), 173-177. Zbl 0707.11032 MR 1019963; Zbl 0712.11026 MR 1079647

[6] R. Brooks, The fundamental group and the spectrum of the Laplacian. Comment. Math. Helv. 56 (1981), 581-598. Zbl 0495.58029 MR 656213

[7] R. Brooks, The spectral geometry of a tower of coverings. J. Differential Geom. 23 (1986), 97-107. Zbl 0576.58033 MR 840402

[8] M. Burger and P. Sarnak, Ramanujan duals II. Invent. Math. 106 (1991), 1-11. Zbl 0774.11021 MR 1123369

[9] J. B. Dennin, Jr., Fields of modular functions of genus 0. Illinois J. Math. 15 (1971), 442-455. Zbl 0223.10010 MR 0304503

[10] J. B. Dennin, Jr., Subfields of $K\left(2^{n}\right)$ of genus 0. Illinois J. Math. 16 (1972), 502-518. Zbl 0255.10029 MR 0306347

[11] A. El Soufi and S. Ilias, Immersions minimales, première valeur propre du laplacien et volume conforme. Math. Ann. 275 (1986), 257-267. Zbl 0675.53045 MR 854009

[12] J. Elstrodt, F. Grunewald, and J. Mennicke, Groups acting on hyperbolic space. Springer Monogr. Math., Springer-Verlag, Berlin 1998, Zbl 0888.11001 MR 1483315

[13] S. Gelbart and H. Jacquet, A relation between automorphic representations of GL(2) and GL(3). Ann. Sci. École Norm. Sup. (4) 11 (1978), 471-542. Zbl 0406.10022 MR 533066

[14] M. L. Gromov and V. A. Rohlin, Embeddings and immersions in Riemannian geometry. Uspehi Mat. Nauk 25 (1970), no. 5(155), 3-62; English transl. Russian Math. Surveys 25 (1970), no. 5 (155), 1-57 Zbl 0202.21004 MR 0290390

[15] J. Hersch, Quatre propriétés isopérimétriques de membranes sphériques homogènes. $C$. R. Acad. Sci. Paris Sér. A 270 (1970), 1645-1648. Zbl 0224.73083 MR 0292357

[16] I. E. Horozov, Euler characteristics of arithmetic groups. Ph.D. thesis, Brown University, Providence, RI, 2003. arXiv:math.GR/0311117

[17] I. E. Horozov, Euler characteristics of arithmetic groups. Math. Res. Lett. 12 (2005), 275-291. Zbl 1114.20025 MR 2150884

[18] H. Jacquet and R. P. Langlands, Automorphic forms on GL(2). Lecture Notes in Math. 114, Springer-Verlag, Berlin 1970. Zbl 0236.12010 MR 0401654

[19] Serge Lang, Algebraic number theory. Addison-Wesley Publishing Co., Reading 1970. Zbl 0211.38404 MR 0282947 
[20] P. Li and S.-T. Yau, A new conformal invariant and its applications to the Willmore conjecture and the first eigenvalue of compact surfaces. Invent. Math. 69 (1982), 269-291. Zbl 0503.53042 MR 674407

[21] D. D. Long, C. Maclachlan, and A. W. Reid, Arithmetic Fuchsian groups of genus zero. Pure Appl. Math. Q. 2 (2006), 569-599. Zbl 1107.20037 MR 2251482

[22] C. Maclachlan and A. W. Reid, The arithmetic of hyperbolic 3-manifolds. Graduate Texts in Math. 219, Springer-Verlag, New York 2003. Zbl 1025.57001 MR 1937957

[23] G. A. Margulis, Discrete groups of motions of manifolds of nonpositive curvature. In Proc. Internat. Congr. Mathematicians (Vancouver, 1974). Vol. 2, Canad. Math. Congress, Montreal 1975, 21-34; English transl. in Amer. Math. Soc. Transl. Ser. (2) 109 (1977), 33-45. Zbl 0336.57037 MR 0492072

[24] V. V. Nikulin, On arithmetic groups generated by reflections in Lobachevsky spaces. Izv. Akad. Nauk SSSR Ser. Mat. 44 (1980), 637-669; English transl. Math. USSR-Izv. 16 (1981), 573-601. Zbl 0441.22008 MR 582161

[25] V. V. Nikulin, On the classification of arithmetic groups generated by reflections in Lobachevsky spaces. Izv. Akad. Nauk SSSR Ser. Mat. 45 (1981), 113-142; English transl. Math. USSR-Izv. 18 (1982), 99-123. Zbl 0477.22008 MR 607579

[26] V. V. Nikulin, Discrete reflection groups in Lobachevsky spaces and algebraic surfaces. In Proc. Internat. Congr. Mathematicians (Berkeley, 1986). Vol. 1, Amer. Math. Soc., Providence, RI, 1987, 654-671. Zbl 0671.22006 MR 0934268

[27] V. V. Nikulin, Finiteness of the number of arithmetic groups generated by reflections in Lobachevsky spaces. Izv. Ross. Akad. Nauk Ser. Mat. 71 (2007), 55-60; English transl. Izv. Math. 71 (2007), 53-56. Zbl 1131.22009

[28] A. M. Odlyzko, Bounds for discriminants and related estimates for class numbers, regulators and zeros of zeta functions: a survey of recent results. Sém. Théor. Nombres Bordeaux (2) 2 (1990), 119-141. Zbl 0722.11054 MR 1061762

[29] V. Platonov and A. Rapinchuk, Algebraic groups and number theory. Pure Appl. Math. 139, Academic Press, Boston 1994. Zbl 0841.20046 MR 1278263

[30] M. N. Prokhorov, Absence of discrete groups of reflections with a noncompact fundamental polyhedron of finite volume in a Lobachevskiü space of high dimension. Izv. Akad. Nauk SSSR Ser. Mat. 50 (1986), 413-424; English transl. Math. USSR-Izv. 28 (1987), 401-411. Zbl 0604.51007 MR 842588

[31] M. Reed and B. Simon, Methods of modern mathematical physics, IV: Analysis of operators. Academic Press, New York 1978. Zbl 0401.47001 MR 0493419

[32] A. A. Ryzhkov and V. I. Chernousov, On the classification of maximal arithmetic subgroups of simply connected groups. Mat. Sb. 188 (1997), no. 9, 127-156; English transl. Sb. Math. 188 (1997), 1385-1413. Zbl 0899.20026 MR 1481667

[33] P. Scott, Subgroups of surface groups are almost geometric. J. London Math. Soc. (2) 17 (1978), 555-565; correction ibid. 32 (1985), 217-220. Zbl 0412.57006 MR 0494062; Zbl 0581.57005 MR 0811778

[34] A. Selberg, On the estimation of Fourier coefficients of modular forms. In Theory of Numbers, Proc. Sympos. Pure Math. 8, Amer. Math. Soc., Providence, R.I., 1965, 1-15. Zbl 0142.33903 MR 0182610 
[35] G. Szegö, Inequalities for certain eigenvalues of a membrane of given area. J. Rational Mech. Anal. 3 (1954), 343-356. Zbl 0055.08802 MR 0061749

[36] W. P. Thurston, Three-dimensional geometry and topology. Vol. 1, Princeton Math. Ser. 35, Princeton University Press, Princeton, NJ, 1997. Zbl 0873.57001 MR 1435975

[37] J. Tits, Classification of algebraic semisimple groups. In Algebraic groups and discontinuous subgroups, Proc. Sympos. Pure Math. 9, Amer. Math. Soc., Providence, R.I., 1966, 33-62. Zbl 0238.20052 MR 0224710

[38] M.-F. Vignéras, Quelques remarques sur la conjecture $\lambda_{1} \geq \frac{1}{4}$. In Seminaire de théorie des nombres, Paris 1981-82, Progr. Math. 38, Birkhäuser Boston, Boston 1983, 321-343. Zbl 0523.10015 MR 0729180

[39] È. B. Vinberg, Discrete groups generated by reflections in Lobačevskiı̌ spaces. Mat. Sb. 72 (114) (1967), 471-488; correction ibid. 73 (115) (1967), 303; English transl. Math. USSR-Sb. 1 (1967), 429-444. Zbl 0166.16303 MR 0207853

[40] È. B. Vinberg, The absence of crystallographic groups of reflections in Lobachevsky spaces of large dimension. Trudy Moskov. Mat. Obshch. 47 (1984), 68-102; English transl. Trans. Moscow Math. Soc. 1985 (1985), 75-112. Zbl 0593.22007 MR 774946

[41] H. C. Wang, Topics on totally discontinuous groups. In Symmetric spaces, Marcel Dekker, New York 1972, 459-487. Zbl 0232.22018 MR 0414787

[42] P. C. Yang and S.-T. Yau, Eigenvalues of the Laplacian of compact Riemann surfaces and minimal submanifolds. Ann. Scuola Norm. Sup. Pisa Cl. Sci. (4) 7 (1980), 55-63. Zbl 0446.58017 MR 577325

[43] P. Zograf, A spectral proof of Rademacher's conjecture for congruence subgroups of the modular group. J. Reine Angew. Math. 414 (1991), 113-116. Zbl 0709.11031 MR 1092625

Received December 12, 2006; revised December 21, 2007

I. Agol, Department of Mathematics, UC Berkeley, 970 Evans Hall \#3840, Berkeley, CA 94720-3840, U.S.A.

E-mail: agol@math.uic.edu

M. Belolipetsky, Department of Mathematical Sciences, Durham University, South Rd, Durham, DH1 3LE, UK

E-mail: mikhail.belolipetsky@durham.ac.uk

P. Storm, Department of Mathematics, David Rittenhouse Lab., 209 South 33rd Street, Philadelphia, PA 19104-6395, U.S.A.

E-mail: storm@math.stanford.edu

K. Whyte, MSCS UIC 322 SEO, m/c 249, 851 S. Morgan St., Chicago, IL 60607-7045, U.S.A.

E-mail: kwhyte@math.uic.edu 